\title{
A Study of Periodic Solution of a Duffing's Equation Using Implicit Function Theorem
}

\author{
E. O. Eze ${ }^{1}$, J. N. Ezeora ${ }^{2}$, U. E. Obasi ${ }^{1}$ \\ ${ }^{1}$ Department of Mathematics, Michael Okpara University of Agriculture, Umuahia, Nigeria \\ ${ }^{2}$ Department of Industrial Mathematics and Statistics, Ebonyi State University, Abakaliki, Nigeria \\ Email: obinwanneeze@gmail.com, sirurchobasi@gmail.com, jerryezeora@yahoo.com, jezeora@aust.edu.ng
}

How to cite this paper: Eze, E.O., Ezeora, J.N. and Obasi, U.E. (2018) A Study of Periodic Solution of a Duffing's Equation Using Implicit Function Theorem. Open Journal of Applied Sciences, 8, 459-464. https://doi.org/10.4236/ojapps.2018.810036

Received: April 23, 2016

Accepted: October 19, 2018

Published: October 22, 2018

Copyright $\odot 2018$ by authors and Scientific Research Publishing Inc. This work is licensed under the Creative Commons Attribution International License (CC BY 4.0).

http://creativecommons.org/licenses/by/4.0/

\begin{abstract}
In this paper, the well known implicit function theorem was applied to study existence and uniqueness of periodic solution of Duffing-type equation. Under appropriate conditions around the origin, a unique periodic solution was obtained.
\end{abstract}

\section{Keywords}

Implicit Function Theorem, Periodic Solution, Duffing Equation, Banach Spaces

\section{Introduction}

The well-known implicit function theorem has been employed by many authors to study existence of solution to non-linear differential equations of various types. [1] [2] [3] investigated the existence of solution to ordinary differential equations using implicit function theorem. Other researchers [4]-[10] used implicit function theorem to show the existence of periodic solution for non-linear partial differential equations. The Duffing equation (oscillator):

$$
\ddot{x}+c \dot{x}+a x+b x^{3}=h(t)
$$

where $a, b, c$ are real constants and $h(t)$ is continuous, has been widely used in physics, economics, engineering, and many other physical phenomena. Given its characteristic of oscillation and chaotic nature, many scientists are inspired by this nonlinear differential equation given its nature to replicate similar dynamics in our natural world. This equation together with Van der Pol's equation has become one of the most common examples of nonlinear oscillation in textbooks and research articles. See for instance [11] [12] [13] [14] and the references 
therein. Due to the importance of the Duffing equation in real world problems, the study of existence of solution of the equation has continued to attract the attention of many researchers. [15] [16] [17] [18] have proposed independently, the existence of periodic solution of Duffing equation of the general form:

$$
\ddot{x}+c \dot{x}+g(t, x)=h(t)
$$

where $h(t)$ is continuous and $2 \pi$-periodic in $t \in R$ and $g(t, x)=a x+b x^{2}+\beta x^{3}$.

Motivated by the above results, the purpose of this paper is to study the existence and uniqueness of periodic solution of Duffing equation of the form:

$$
\ddot{x}+c \dot{x}+a x+b x^{2}+2 x^{3}=h(t)
$$

using implicit function theorem where $a, b, c$ are real constants and $h:[0,2 \pi] \rightarrow R^{n}$ is continuous with boundary conditions

$$
\begin{aligned}
& x(0)=x(2 \pi) \\
& \dot{x}(0)=\dot{x}(2 \pi)
\end{aligned}
$$

\section{Preliminaries}

Definition 2.1. Consider the general non-linear differential equation of the form

$$
\dot{x}=f(t, x(t))
$$

where $f: \mathbb{R} \times \mathbb{R}^{n} \rightarrow \mathbb{R}^{n}$ is continuous. The function $f$ in Equation (2.1) is said to be $T$ periodic if for every $(t, x) \in R \times R^{n}$ and some $T>0 . f(t, x)=f(t+T, x)$ and $f(t, x) \neq f\left(t+T^{*}, x\right)$ for all $T^{*}<T$.

Definition 2.2. A solution $x$ of Equation (2.1) defined on $R$ such that $x(t+T)=x(t)$ for all $t \in R$ is called $T$ periodic solution or $T$ periodic solution.

Definition 2.3. Let $E, F$ be Banach spaces, $U$ an open subset of $E$ and let $x_{0} \in U$. Let $f: U \rightarrow F$ be a mapping of $U$ into $F$. $f$ is said to be Frechet differentiable at $x_{0}$ if there exists a continuous linear mapping; $L: E \rightarrow F$ such that

$$
\lim _{x \rightarrow x_{0}} \frac{\left\|f(x)-f\left(x_{0}\right)-L\left(x-x_{0}\right)\right\|}{\left\|x-x_{0}\right\|}=0
$$

Definition 2.4. Let $E, F, G$ be Banach spaces, let $U=u_{1} \times u_{2} \in E \times F$ be open set and $f: U \rightarrow F$ be a mapping of $U$ into $G$, with $\left(x_{0}, y_{0}\right) \in U$. $f$ is said to be Frechet differentiable with respect to the first variable $x$ at $\left(x_{0}, y_{0}\right)$ if the following conditions hold.

1) There exists a continuous linear mapping $L_{1}: E \rightarrow G$ such that $\forall \varepsilon>0$ $\exists \delta>0$ such that $\forall x, \bar{x} \in u_{1}$ then $\left\|x-x_{0}\right\| \leq \delta,\left\|\bar{x}-x_{0}\right\| \leq \delta$ and $\forall y \in u_{2}$ such that $\left\|y-y_{0}\right\| \leq \delta$, it follows that.

2) $\left\|f(x, y)-f(\bar{x}, y)-L_{1}(x-\bar{x})\right\| \leq \varepsilon\|x-\bar{x}\|$.

3) The mapping $y \rightarrow f\left(x_{0}, y\right)$ is continuous at $y_{0}$.

Proposition 2.5. Then condition (1) of the definition 2.4 is satisfied if the 
partial Frechet derivative $f_{1}^{1}\left(x_{0}, y_{0}\right)$ exists for $(x, y)$ in a neighbourhood of $\left(x_{0}, y_{0}\right)$ and if the mapping $(x, y) \rightarrow f_{1}^{1}\left(x_{0}, y_{0}\right)$ is continuous at $\left(x_{0}, y_{0}\right)$.

Proposition 2.6. If $f$ is Frechet differentiable with respect to the first variable at $\left(x_{0}, y_{0}\right)$, it is Frechet differential with respect to this variable at $\left(x_{0}, y_{0}\right)$ with the same $L_{1}$. Moreover, this is unique. $L_{1}$ is called the strong partial Frechet derivative with respect to the first variable at $\left(x_{0}, y_{0}\right)$ and denoted by $f_{1}^{1}\left(x_{0}, y_{0}\right)$.

Lemma 2.7. (The Banach fixed point theorem) Let $E$ be a Banach space and $f: E \rightarrow E$ be a contraction mapping, then $f$ has a unique fixed point in $E$, i.e. there exists a unique $x \in E$ such that $f(x)=x$.

Lemma 2.8. (The implicit function theorem) Let $E, F, G$ be Banach spaces and let $U_{1} \subset E, U_{2} \subset F$. Set $U=U_{1} \times U_{2}$. For arbitrary $\left(x_{0}, y_{0}\right) \in U$, let $f: U \rightarrow G$ be a mapping satisfying the following conditions.

1) $f\left(x_{0}, y_{0}\right)=0$.

2) $f$ is Frechet differentiable with respect to the first variable at $\left(x_{0}, y_{0}\right)$.

3) $f_{1}^{\prime}\left(x_{0}, y_{0}\right): E \rightarrow G$ is a linear homeomorphism.

Then there exists a neighborhood $V_{1} \times V_{2} \subset U_{1} \times U_{2}$ of $\left(x_{0}, y_{0}\right)$ and a unique mapping $\zeta: V_{1} \rightarrow V_{2}$ such that for each $y \in V_{2}$ the equation $f(x, y)=0$ has in $V_{1}$, the unique solution $f(\zeta(y), y)=0$ : Moreover, $\zeta$ is continuous at $y_{0}$.

Lemma 2.9. If $X$ and $Y$ are Banach spaces and $A \in B(X, Y)$ with $R(A)=Y$ and $N(A)=\{0\}$, then $A^{-1} \in B(Y, X)$ where $N(A)$ is the Null spaces of $A$ and $R(A)$ is the range space of $A . B(X, Y)$ is the space of bounded linear transformations from $X$ to $Y$.

\section{Main Result}

We present in this section, the main result of this paper.

Theorem 3.1. Let $C_{2 \pi}^{2}=\left\{x:[0, T] \rightarrow R^{n}: x\right.$ is a class of $\left.C^{2}\right\}$ and equipped with the usual uniform norm $\|x\|_{\infty}=\max \{|x(t)|,|\dot{x}(t),| \ddot{x}(t) \mid\} \quad C=\{x:[0, T] \rightarrow \mathbb{R}$ : $x$ is continous $\}$ with the usual norm, $J=[0, T], \quad J_{2 \pi}=[0,2 \pi]$.

Then, Equation (1.2) is equivalent to

$$
f(x, h)=0 \text { in } C_{2 \pi}^{2} \times C
$$

where $f: C_{2 \pi}^{2} \times C \rightarrow C$ is defined by

$$
f(x, h)=\ddot{x}+c \dot{x}+a x+b x^{2}+2 x^{3}-h
$$

Proof: We first remark that with the norm defined above, $C_{2 \pi}^{2}$ is a Banach space. The strategy for the proof involves application of the implicit function theorem to the function $f$ defined in Equation (3.1). We split the proof into steps.

Step 1: $f(0,0)=0$. This follows trivially from the definition of $f$ : hence

$$
f(0, h)=-h
$$

Step 2: $f$ is Frechet differentiable with respect to $x$ at $(0 ; 0)$. Observe that 


$$
\begin{aligned}
& \left|f(x, h)-f(\bar{x}, h)-f_{x}(0,0)(x, \bar{x})\right| \\
& =\left|\ddot{x}+c \dot{x}+a x+b x^{2}+2 x^{3}-h-\left(\ddot{\bar{x}}+c \dot{\bar{x}}+a \bar{x}+b \bar{x}^{2}+2 \bar{x}^{3}-h\right)\right| \\
& =\left|(\ddot{x}-\ddot{\bar{x}})+c(\dot{x}-\dot{\bar{x}})+a(x-\bar{x})+b\left(x^{2}-\bar{x}^{2}\right)+2\left(x^{3}-\bar{x}^{3}\right)\right| \\
& \leq|x-\bar{x}| \cdot\left|b(x+\bar{x})+2\left(x^{2}+x \bar{x}+\bar{x}^{2}\right)\right| \\
& \leq|x-\bar{x}| b(|x|+|\bar{x}|)+2\left|\left(x^{2}+x \bar{x}+\bar{x}^{2}\right)\right| \\
& \leq|x-\bar{x}| b(|x|+|\bar{x}|)+2\left(|x|^{2}+|x \bar{x}|+|\bar{x}|^{2}\right) \\
& \quad \leq|x-\bar{x}|\left(2 b p+6 p^{2}\right), \text { with }|x| \leq p,|\bar{x}| \leq p
\end{aligned}
$$

Consequently,

$$
\left|f(x, h)-f(\bar{x}, h)-f_{x}(0,0)(x, \bar{x})\right| \rightarrow 0, \text { as }|x-\bar{x}| \rightarrow 0
$$

Combining (3.3) and (3.4), we obtain that $f$ is Frechet differentiable with respect to the first variable at $(0,0)$.

Step 3: $f_{x}(0,0): C_{2 \pi}^{2} \rightarrow C$ defined by $z \rightarrow \ddot{z}+c \dot{z}+a z$ is a linear homeomorphism.

The mapping $f_{x}(0,0): z \rightarrow \ddot{z}+c \dot{z}+a z$ is linear and continuous and hence bounded. It is also an onto mapping. Linear homeomorphism would have been established if the mapping is shown to be one to one. This is equivalent to requiring that

$$
\ddot{z}+c \dot{z}+a z=0
$$

with

$$
z(0)=z(2 \pi) \text { and } \dot{z}(0)=\dot{z}(2 \pi)
$$

be non-critical.

It suffices to place appropriate conditions on the constants $a, c$ such that Equation (3.5) is solvable. The auxiliary equation of (3.5) is $\lambda^{2}+c \lambda+a=0$.

Case I:

If $c=0$, and $a=k^{2}$ where $k$ is a natural number, then $\lambda= \pm i k$ and

$$
z(t)=c_{1} \cos k t+c_{2} \sin k t
$$

for arbitrary constants $c_{1}$ and $c_{2}$. Clearly

$$
z(0)=z(2 \pi) \text { and } \dot{z}(0)=\dot{z}(2 \pi)
$$

and the solution is non-trivial.

Case II:

If $c=0$ and $a \neq k^{2}$, then condition

$$
\dot{z}(0)=\dot{z}(2 \pi)
$$

is satisfied only by the trivial solution $z=0$.

Case III:

If $c \neq 0$ and $a \neq 0$, only the trivial solution exists. Most generally, put

$$
\lambda=\frac{-c \pm \sqrt{c^{2}-4 a}}{2}=u+i v
$$


for some real numbers $u, v$.

1) Choose $c$ and a such $u \neq 0$.

2) Choose $c$ and a such that $u \neq 0, v=0$ then $z \rightarrow \ddot{z}+c \dot{z}+a z$ is non-critical [19].

3) Choose $c$ and a such that $I-x(2 \pi)$ is non-singular where $x(t)$ is fundamental matrix of Equation (3.5) with $x(0)=I$ the $2 \times 2$ identity matrix [20].

Thus with any of these conditions imposed, one deduces the one to oneness of $f_{x}^{\lfloor}(0,0)$. Hence by Lemma $2.8\left[f_{x}^{\lfloor}(0,0)\right]^{-1}$ exists as a bounded linear operator. Linear homeomorphism of $f_{x}^{\dagger}(0,0)$ follows. Existence of a unique solution is now assured by the implicit function theorem.

\section{Conflicts of Interest}

The authors declare no conflicts of interest regarding the publication of this paper.

\section{References}

[1] Chow, S.N. and Lasota, A. (1972) An Implicit Function Theorem for Non-Differentiable Mappings. Proceedings of the American Mathematical Society, 34, 141-146. https://doi.org/10.1090/S0002-9939-1972-0291527-7

[2] Chicone, C. (1996) Ordinary Differential Equation with Applications. Springer-Verlag, New York.

[3] Hartman, P. (2014) Ordinary Differential Equation. 2nd Edition, Willey and Sons Publications, New York, 235-239.

[4] Kreici, P. (1984) Hard Implicit Function Theorem and Small Periodic Solutions to Partial Differential Equations. Commentationes Mathematicae Universitatis Carolinae, 25, 519-536.

[5] Magnus, R. (1974) The Implicit Function Theorem and Multi Bump Solutions of periodic Partial Differential Equation. Proceedings of the Royal Society of Edinburgh Section A: Mathematics, 136, 559-583.

[6] Mishra, L.N. and Sen, M. (2016) On the Concept of Existence and Local Attractivity of Solutions for Some Quadratic Volterra Integral Equation of Fractional Order. Applied Mathematics and Computation, 285, 174-183.

[7] Mishra, L.N., Agarwal, R.P. and Sen, M. (2016) Solvability and Asymptotic Behavior for Some Nonlinear Quadratic Integral Equation Involving Fractional Integrals on the Unbounded Interval. Progress in Fractional Differentiation and Applications, 2, 153-168.

[8] Deepmala (2014) A Study on Fixed Point Theorem for Nonlinear Contraction and Its Applications. Ph.D. Thesis, Pt. Ravishankar Shukla University, Raipur, India.

[9] Mishra, V.N. (2007) Some Problems on Approximations of Functions in Banach Spaces. Ph.D. Thesis, Indian Institute of Technology, Roorkee, India.

[10] Deepmala and Pathak, H.K. (2013) A Study on Some Problems on Existence of Solutions for Nonlinear Functional-Integral Equation. Acta Mathematica Scientia, 33, 1305-1313.

[11] Puu, T. (2000) Attractors, Bifurations \& Chaos: Nonlinear Phenomena in Economics. Spring-Verlag, Berlin, Heidelberg. 
[12] Ueda, Y. (1979) Randomly Transitional Phenomena in the System Governed by Duffing's Equation. Journal of Statistical Physics, 20, 181-196. https://doi.org/10.1007/BF01011512

[13] Chen, H.B. and Li, Y. (2000) Existence, Uniqueness and Stability of Periodic Solution of an Equation of Duffing Type. AIMS Journal, 10, 10-20.

[14] Zhang, W.B. (2005) Differential Equations, Bifurcations, and Chaos in Economics. World Scientific, Singapore. https://doi.org/10.1142/5827

[15] Njoku, F.I. and Omari, P. (2003) Stability Properties of Periodic Solutions of a Duffing's Equation in the Presence of a Lower and Upper Solutions. Applied Mathematics and Computation, 135, 471-490. https://doi.org/10.1016/S0096-3003(02)00062-0

[16] Pedro, J.T. (2004) Existence and Stability of Periodic Solutions of a Duffing's Equation by Using a New Maximum Principle. Mediterranean Journal of Mathematics, 1, 470-486.

[17] Sani, G. and Alain, H.N. (1989) N-Cyclic Function and Multiple Subharmonic Solutions of Duffing's Equation.

[18] Yuji, L. and Weiguo, G. (2004) Positive Solution of Non-Linear Duffing's Equation with Delay and Variable Coefficients. Tamsu Oxford Journal of Mathematical Sciences, 20, 235-255.

[19] Hale, J.K. (1963) Oscillation in Non-Linear System. McGraw Hill, New York.

[20] Hale, J. and Taboas, P.Z. (1978) Interaction of Forced Damping in a Second Order Evolution Equation. 